Communications in Physics, Vol. 28, No. 4 (2018), pp. 361-367

DOI:10.15625/0868-3166/28/4/13182

\title{
COMPETITION BETWEEN THE SOFT GAP AND THE MOLECULAR KONDO SINGLETS IN FLAT-BAND LATTICES
}

\author{
DUONG-BO NGUYEN ${ }^{1, \dagger}$, HONG-SON NGUYEN $^{2}$ AND MINH-TIEN TRAN ${ }^{1,3}$ \\ ${ }^{1}$ Graduate University of Science and Technology, \\ Vietnam Academy of Science and Technology, Hanoi, Vietnam \\ ${ }^{2}$ Department of Occupational Safety and Health, \\ Trade Union University, 169 Tay Son, Hanoi, Vietnam \\ ${ }^{3}$ Institute of Physics, \\ Vietnam Academy of Science and Technology, Hanoi, Vietnam \\ ${ }^{\dagger} E$-mail: nguyenduongbo80@gmail.com \\ Received 07 October 2018 \\ Accepted for publication 04 November 2018 \\ Published 15 December 2018
}

\begin{abstract}
The Kondo problem of a magnetic impurity embedded in the Lieb lattice is studied by the numerical renormalization group. The magnetic impurity hybridizes with conduction electrons from both the flat-and the soft-gap bands. We find a competition between the soft gap and the molecular Kondo singlet formations. The molecular Kondo effect occurs only when the magnetic impurity strongly hybridizes with conduction electrons at edge center sites of the Lieb lattice, and at the temperature range between the artificial strong coupling and the local moment regimes.
\end{abstract}

Keywords: Kondo effect, numerical renormalization group, strongly correlated electron systems, flat band, soft gap.

Classification numbers: 71.27.+a, 72.15.Qm, 75.20.Hr, 71.10.Fd.

\section{INTRODUCTION}

Electron correlations in flat-band lattices have attracted a lot of research attention due to their special features. In flat-band lattices, the Coulomb interaction of electrons becomes dominant over the kinetic energy, and as a consequence intriguing phenomena such as the flat-band ferromagnetism or the molecular Kondo effect emerge [1,2]. When a magnetic impurity is coupled to conduction electrons from the flat band, it forms the molecular Kondo singlet with a single conduction electron and quenches all other conduction electrons in the Kondo singlet formation [2-5]. This essentially yields a pure two spin qubits in solids, where the band flatness exists. However, in (C)2018 Vietnam Academy of Science and Technology 
flat-lattice lattices, in addition to the band flatness, conduction electrons of other bands often have special low-energy properties, for instance, in the Lieb lattice, conduction electrons also exhibit the Dirac cones nearby the corners of the first Brillouin zone [1,2]. When a magnetic impurity is coupled to such conduction electrons, they together yield a soft-gap Kondo problem [6,7]. In this way, the flat-band lattices would allow us to study the competition between the soft-gap and the molecular Kondo strong coupling when the magnetic impurity is coupled with both the flat-band and soft-gap band conduction electrons. For this purpose we will study the Kondo problem in the Lieb lattice. The Lieb lattice is the two-dimensional edge-centered square lattice [1,2]. It is one of the simplest flat-band lattices, and is often used to study the effects of the band flatness [1]. The electron structure of the Lieb lattice exhibits both the band flatness and soft gap [1,2]. In contrast to the previous study, where the magnetic impurity is coupled only to the flat-band conduction electrons [2], in this present work the magnetic impurity is coupled to both the flat band and soft-gap conduction electrons. This can be achieved by extending the local hybridization between the magnetic impurity and conduction electrons to nonlocal ones. The local hybridization of the magnetic impurity at the square corner site of the Lieb lattice yields the soft gap Kondo problem, while the nonlocal hybridization between the magnetic impurity at the corner site and conduction electrons at the nearest neighbor sites essentially yields the molecular Kondo problem [2]. In such the way we are able to study the competition between the soft gap and molecular Kondo singlet in the Lieb lattice. We will use the numerical renormalization group (NRG) to solve the proposed Kondo problem [7-10]. The NRG was essentially introduced to solve the Kondo problem in metals [7-10]. It accurately describes all possible regimes in the Kondo problem [7-10]. The NRG has also been extended to solve the Kondo problem in different environments, such as in softgap systems, superconductors,... [7]. Recently, the NRG was successfully applied to the Kondo problem in the flat-band systems [2].

The organization of the present paper is as follows. In Sec. II we present the model. The NRG calculations and their results are presented in Sec. III. Section IV is the conclusion.

\section{MODEL}

One of the simplest flat-band lattices is the Lieb lattice [1]. The Lieb lattice is a square lattice with additional sites at the middle of every square edge (see Fig. 1). The Lieb lattice has attracted research attention since the discovery of high-temperature superconductivity because it is the basic structure of $\mathrm{CuO}_{2}$ plane of the cuprate superconductors [1]. The Lieb lattice can be also artificially made by optical lattices [11], ultracold atoms [12], and molecular design [13].

We consider a magnetic impurity, which is placed at a corner site (A site in Fig. 1), and hybridizes with conduction electrons at site $\mathrm{A}$ as well as at the nearest neighbor sites. The Hamiltonian of the model reads

$$
\begin{aligned}
H= & -t \sum_{\langle i, j\rangle, \sigma} c_{i \sigma}^{\dagger} c_{j \sigma}+\varepsilon \sum_{\sigma} n_{A \sigma}^{f}+U n_{A \uparrow}^{f} n_{A \downarrow}^{f} \\
& +V_{0} \sum_{\sigma} c_{A \sigma}^{\dagger} f_{A \sigma}+H . c .+V_{1} \sum_{i(A), \sigma} c_{i \sigma}^{\dagger} f_{A \sigma}+H . c .,
\end{aligned}
$$

where $c_{i \sigma}^{\dagger}\left(c_{i \sigma}^{)}\right.$is the creation (annihilation) operator of conduction electron at site $i$ with spin $\sigma . t$ is the hopping parameter. Here we take into account only the nearest neighbor hopping 


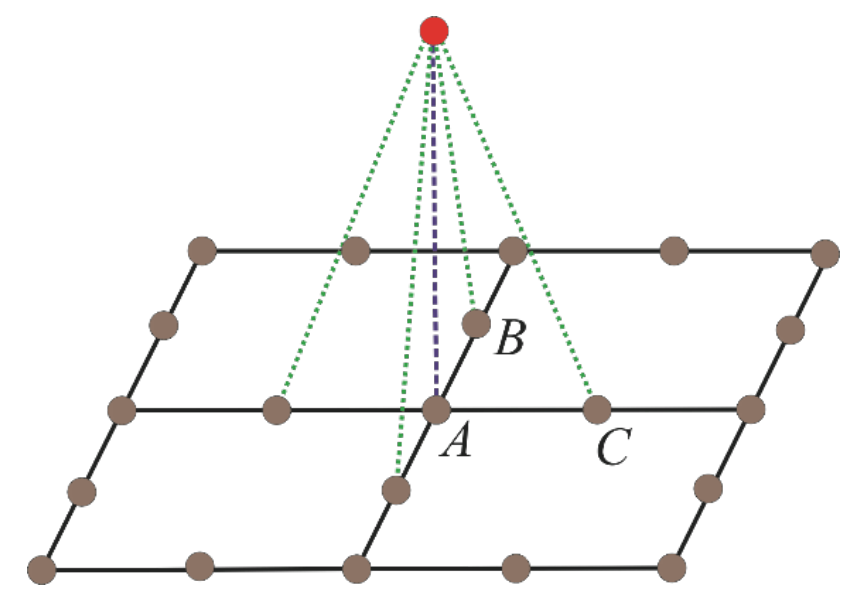

Fig. 1. The Lieb lattice structure. The magnetic impurity (red dot) is hybridized with conduction electrons at the corner site (A site) as well as at the edge center sites, surrounding the corner site (B and $\mathrm{C}$ sites).

of conduction electrons. $f_{A \sigma}^{\dagger}\left(f_{A \sigma}\right)$ is the creation (annihilation) operator of impurity with spin $\sigma$ at site $A . \quad n_{A \sigma}^{f}=f_{A \sigma}^{\dagger} f_{A \sigma}$ is the number operator of the magnetic impurity. $\varepsilon$ is the energy level of the magnetic impurity, and $U$ is the Coulomb interaction of electrons at the impurity site. The magnetic impurity hybridizes with conduction electrons at site $A$ by strength $V_{0}$, and with conduction electrons at nearest neighbor sites by strength $V_{1}$. When $V_{1}=0$, Hamiltonian in Eq. (1) reduces to the one of the soft-gap Kondo problem [6,7]. When $V_{0}=0$, Hamiltonian in Eq. (1) basically describes the molecular Kondo problem [2]. For finite hybridizations $V_{0}, V_{1}$, Hamiltonian in Eq. (1) would describe the competition between the soft-gap and the molecular Kondo effect, which may occur in the system. We parameterize these hybridization strengths by

$$
\begin{aligned}
& V_{0}=\frac{1}{2}(1+\alpha) V, \\
& V_{1}=\frac{1}{2}(1-\alpha) V .
\end{aligned}
$$

When $\alpha=1, V_{0}=V$ and $V_{1}=0$. When $\alpha=-1, V_{0}=0$ and $V_{1}=V$. When $-1<\alpha<1$, both $V_{0}$ and $V_{1}$ are finite. The parameter $\alpha$ describes the difference relation between $V_{0}$ and $V_{1}$, while $V$ is the total hybridization strength.

The Kondo problem totally depends on the hybridization function [7-10]

$$
\Delta(\omega)=\frac{1}{N} \sum_{k} \Gamma_{k}^{\dagger}\left[\omega-h_{0}(k)\right]^{-1} \Gamma_{k},
$$

where $\Gamma_{k}^{\dagger}=\left(V_{0}, 2 V_{1} \cos \left(k_{x} / 2\right), 2 V_{1} \cos \left(k_{y} / 2\right)\right)$, and $h_{0}(k)$ is the Bloch Hamiltonian of conduction electrons

$$
h_{0}(k)=\left(\begin{array}{ccc}
0 & -2 t \cos \left(k_{x} / 2\right) & -2 t \cos \left(k_{y} / 2\right) \\
-2 t \cos \left(k_{x} / 2\right) & 0 & 0 \\
-2 t \cos \left(k_{y} / 2\right) & 0 & 0
\end{array}\right)
$$




\section{NUMERICAL RENORMATION GROUP RESULTS}

We use the NRG to solve the Kondo problem in the Lieb lattice, which is described by Hamiltonian in Eq. (1). The NRG Ljubjana package is used [14-16]. In numerical calculations the half band width $D=2 \sqrt{2} t=1$ is used as the energy unit. We set $\Gamma=\pi V^{2}$, and consider only the symmetric case $\varepsilon=U / 2$. In the NRG the logarithm mesh is discretized by the $\mathrm{Z}$ scheme with the energy cutoff $\Lambda=2$ [14-16]. All calculated quantities are averaged over $N_{z}=8$ interleaved logarithm meshes with the twist parameter $z=1 / N_{z}$ [14-16]. The impurity thermodynamical quantity $O$ is defined as $O_{i m p}=O_{t o t}-O_{t o t}^{(0)}$, where $O_{t o t}$ is the thermodynamical quantity of the total system, and $O_{t o t}^{(0)}$ is the one without the impurity [7]. This is the contributions of the magnetic impurity to the thermodynamical quantity. Like the previous study, in order to take into account the flat-band feature we broaden the flat band by a small positive quantity $\eta$, i.e. replace $\Delta(\omega) \rightarrow$ $\Delta(\omega+i \eta)[2]$.

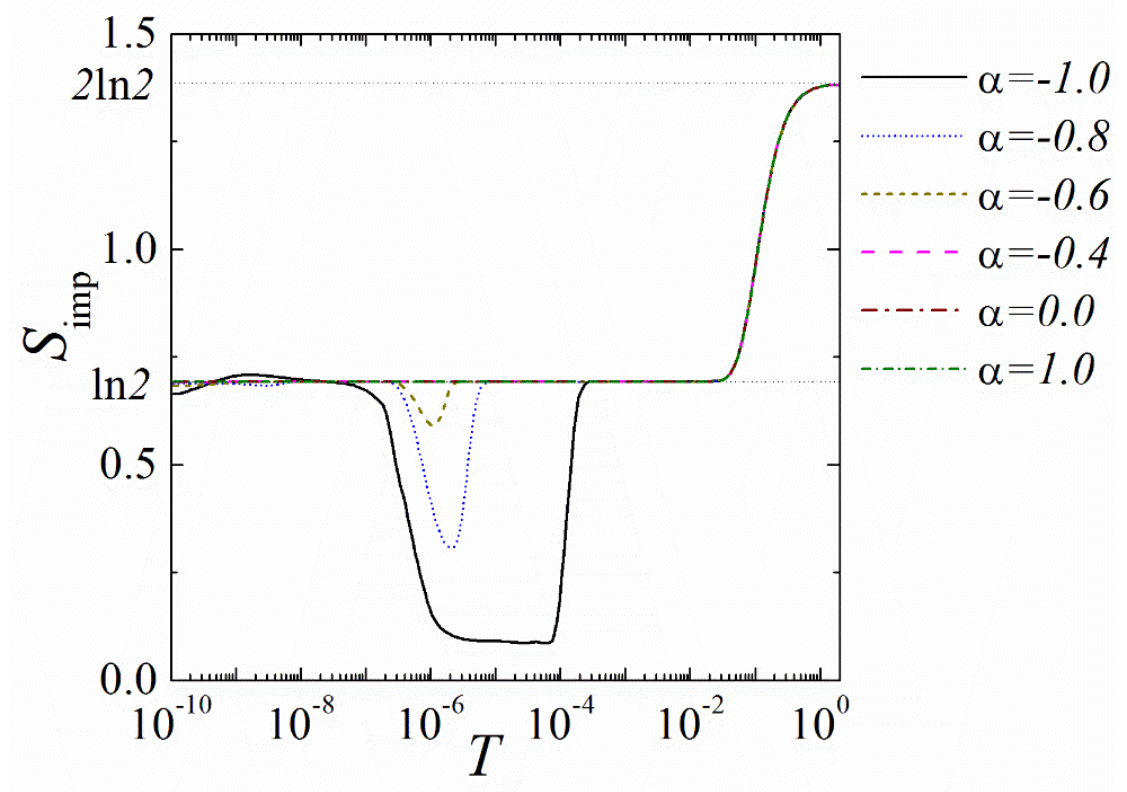

Fig. 2. The impurity entropy as a function of temperature for different values of $\alpha$. $U=$ $0.5, \Gamma=0.001, \eta=10^{-9}$.

In Fig. 2 we plot the impurity entropy for different parameters $\alpha$. When $\alpha=1$, the impurity entropy shows two regimes. At low temperature $S_{i m p}=\ln 2$. This low temperature regime is the local moment (LM) one. The magnetic impurity behaves as a local moment, therefore $S_{i m p}=$ $\ln 2$ [7-10]. When temperature increases, the impurity entropy increases toward to the free orbital (FO) entropy value $2 \ln 2$. The high temperature regime is the FO one [7-10]. The case $\alpha=1$ is the soft gap Kondo problem, as expected, because $\alpha=1$ implies $V_{0}=V$ and $V_{1}=0$. In this case the magnetic impurity is coupled only to conduction electrons at $A$ site. Since conduction electrons at $A$ site exhibit the soft-gap feature, hence the Kondo problem is the soft-gap one [6,7]. When $\alpha$ decreases towards to the opposite limit $\alpha=-1$ a novel regime, which occurs at temperature lower 
than the LM regime, appears. In this regime, the impurity entropy is smaller than the LM one. It decreases towards the value of the impurity entropy in the limit $\alpha=-1$. When $\alpha=-1, V_{0}=0$ and $V_{1}=V$ imply the magnetic impurity is coupled to conduction electrons at the edge center sites. The local density of states of conduction electrons at the edge center sites exhibits both the softgap and the flat-band features. This yields the molecular Kondo problem, where the flat-band conduction electrons play a dominant role in the Kondo singlet formation [2]. The conduction electrons of the soft-gap bands are irrelevant to the Kondo-singlet formation [2]. However, in contrast to the previous study [2], the hybridization in the case $\alpha=-1$ is momentum dependent. Due to this feature, the impurity entropy does not approach to the value of the impurity entropy in the molecular Kondo effect [2]. At sufficient low temperature, typically bellows $\eta$, a strong coupling regime appears. However, this regime is artificial, due to the finite value of $\eta$. In the limit $\eta \rightarrow 0$, it disappears. In addition, in this regime, the impurity entropy fluctuates around the entropy value of the LM regime.

Like in the previous study [2], the NRG results depend on the broadening parameter $\eta$, as shown in Fig. 3. In figure 3 we plot the temperature dependence of the impurity entropy for different values of $\eta$. We consider the case $\alpha=-1$, because in this case, the effect of the flat band is strongest, as it is shown in Fig. 2. One can see in Fig. 3, in the LM and the FO regimes, the impurity entropy is independent on $\eta$. However, in the molecular Kondo effect regime, the impurity entropy depends on $\eta$, as expected, because the width of the flat band influences on the Kondo singlet formation.

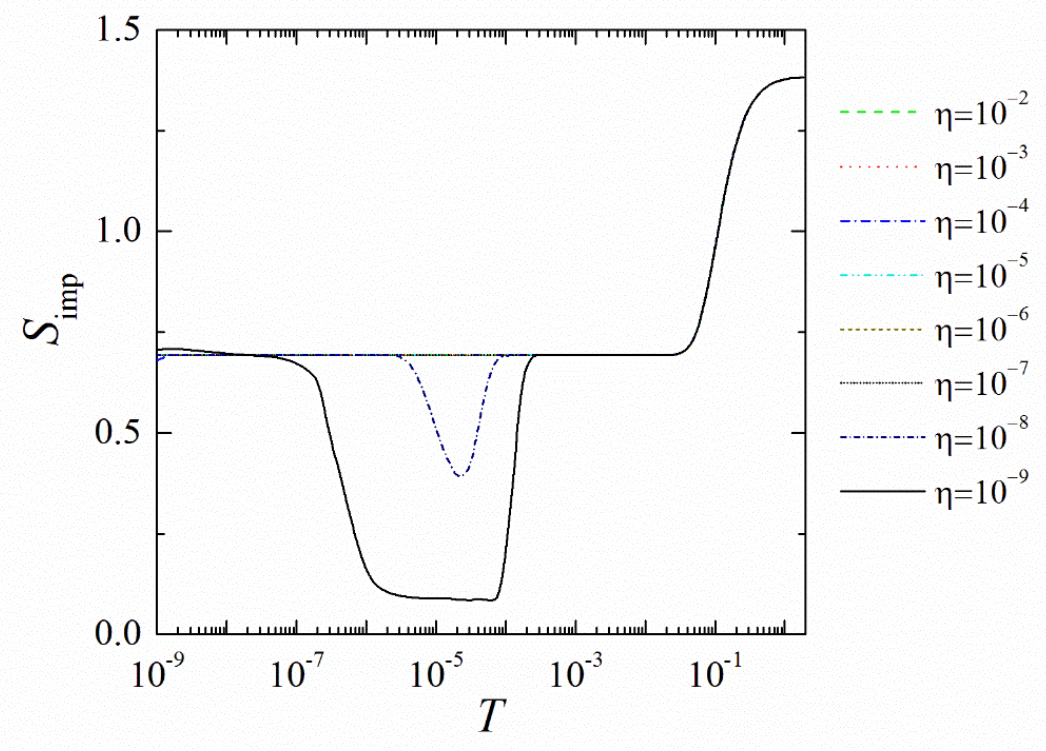

Fig. 3. The impurity entropy as a function of temperature for different values of $\eta$. $U=$ $0.5, \Gamma=0.001, \alpha=-1$.

Similarly, the impurity spin susceptibility also depends on the broadening parameter $\eta$, as shown in Fig. 4. In Fig. 4 we plot the temperature dependence of the impurity spin susceptibility for different values of $\eta$. In the LM and the FO regimes, the impurity spin susceptibility again 
does not depend on $\eta$, providing it is small. However, in the regime of the molecular Kondo effect, the impurity spin susceptibility strongly depends on $\eta$.

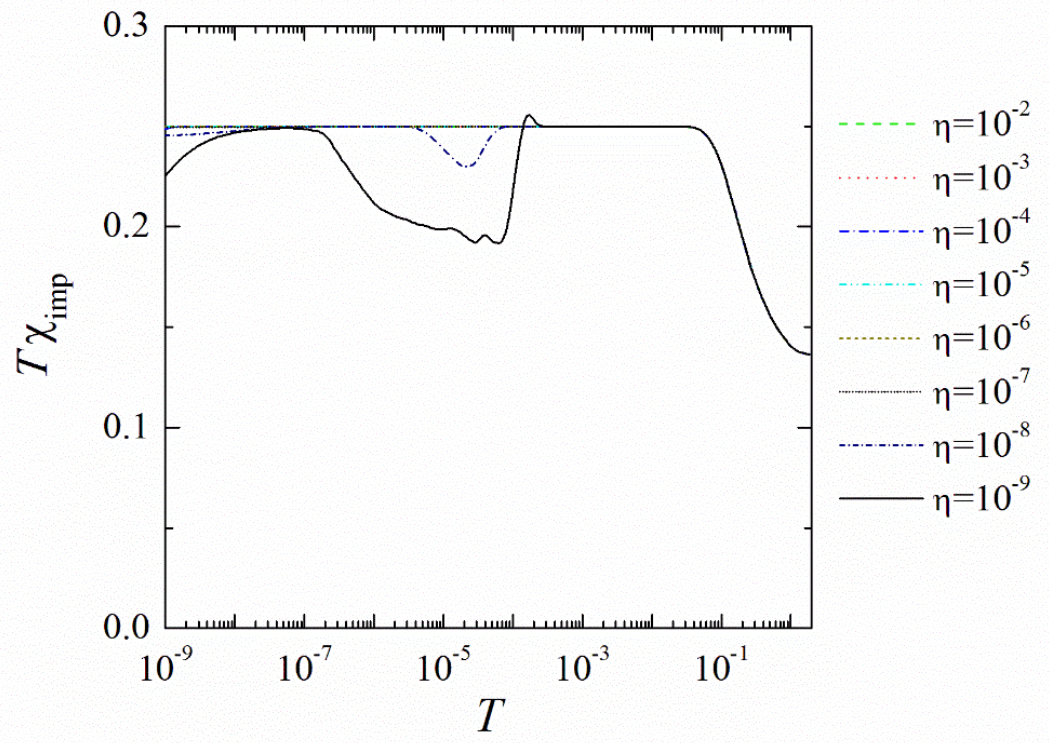

Fig. 4. The impurity spin susceptibility as a function of temperature for different values of $\eta . U=0.5, \Gamma=0.001, \alpha=-1$.

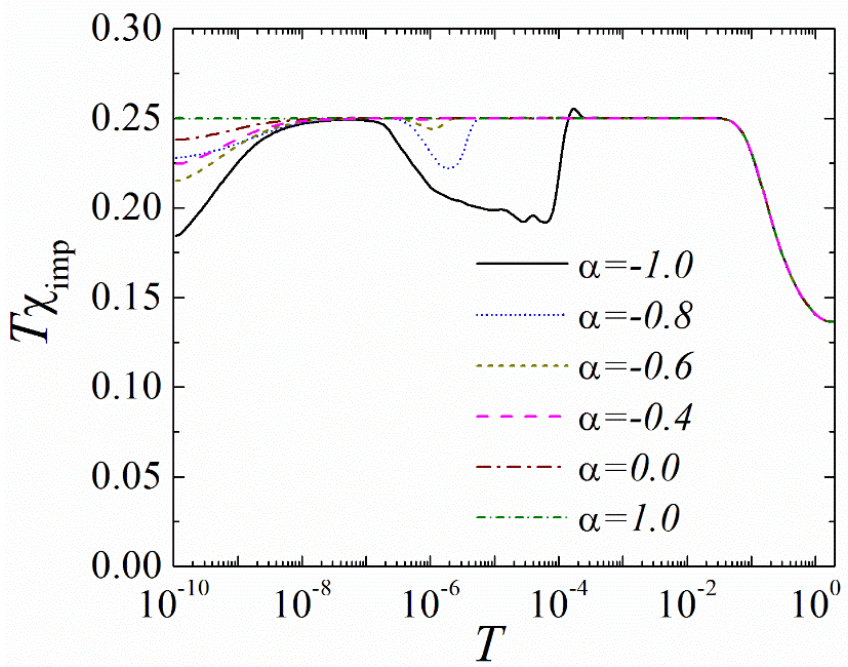

Fig. 5. The impurity spin susceptibility as a function of temperature for different values of $\alpha . U=0.5, \Gamma=0.001, \eta=10^{-9}$.

In Fig. 5 we present the temperature dependence of the impurity spin susceptibility for different values of $\alpha$. In the limit $\alpha=1$, one can see again the soft-gap Kondo problem. There 
are only two regimes: LM and FO. In the LM regime $T \chi_{i m p}=1 / 4$, and in the FO regime it approaches to $1 / 8$ [7-10]. The molecular Kondo effect appears only when $\alpha<0$. This condition implies $V_{1}>V_{0}$, i. e., the impurity hybridization with conduction electrons at the edge center sites (B and $\mathrm{C}$ sites) must be stronger than the one with conduction electrons at the corner site (A site). The molecular Kondo effect is strongest when $\alpha=-1$, i.e, when the magnetic impurity is decoupled from the conduction electrons at the corner site. However, due to the momentum dependence of the magnetic impurity hybzidization with conduction electrons at the edge center sites, the impurity spin susceptibility does not approach to $-1 / 8$, as in the case of momentum independent of hybridization [2]. At sufficient low temperature, typically $T<\eta$, the system goes to the strong coupling due to the finite value of $\eta$. However, as we have mentioned, this regime is artificial due to finite value of $\eta$. In the limit $\eta \rightarrow 0$, it disappears. The properties of the impurity susceptibility is consistent with the behaviors of the impurity entropy in Fig. 2.

\section{CONCLUSION}

We have studied the Kondo problem in the Lieb lattice when the magnetic impurity hybridizes with conduction electrons of both the flat- and the soft-gap bands. The Kondo effect strongly depends on the relation of the hybridizations at the corner site and at the surrounding edge-center sites. When the hybridization at the corner site is stronger, only the LM and FO regimes exist. The molecular Kondo regime appears only when the hybridizations at the edgecenter sites are stronger, and below the molecular Kondo temperature. It occurs as a result of the competition between the soft-gap and the molecular Kondo effects in the Lieb lattice.

\section{ACKNOWLEDGMENTS}

This research is funded by Vietnam National Foundation for Science and Technology Development (NAFOSTED) under Grant No 103.01-2017.13.

\section{REFERENCES}

[1] H. Tasaki, Prog. Theor. Phys. 99 (1998) 489.

[2] Minh-Tien Tran and Thuy Thi Nguyen, Phys. Rev. B 97 (2018) 155125.

[3] P. Fulde, Electron Correlations in Molecules and Solids, Springer Series in Solid-State Science, 3rd ed., Springer (2002).

[4] C.-S. Neumann and P. Fulde, Z. Phys. B 74 (1989) 277.

[5] E. Runge and G. Zwicknagl, Ann. Phys. 508 (1996) 333.

[6] L. Fritz and M. Vojta, Rep. Prog. Phys. 76 (2013) 032501.

[7] R. Bulla, T. A. Costi, and T. Pruschke, Rev. Mod. Phys. 80 (2008) 395.

[8] K. G. Wilson, Rev. Mod. Phys. 47 (1975) 773.

[9] H. R. Krishna-murthy, J. W. Wilkins, and K. G. Wilson, Phys. Rev. B 21 (1980) 1003.

[10] H. R. Krishna-murthy, J. W. Wilkins, and K. G. Wilson, Phys. Rev. B 21 (1980) 1044.

[11] S. Mukherjee, A. Spracklen, D. Choudhury, N. Goldman, P. Ohberg, E. Andersson, and R. R. Thomson, Phys. Rev. Lett. 114 (2015) 245504.

[12] S. Taie, H. Ozawa, T. Ichinose, T. Nishio, S. Nakajima and Y. Takahashi, Science Adv. 1 (2015) 1500854.

[13] M. R. Slot, T. S. Gardenier, P. H. Jacobse, G. C. P. van Miert, S. N. Kempkes, S. J. M. Zevenhuizen, C. M. Smith, D. Vanmaekelbergh, and I. Swart, Nat. Phys. 13 (2017) 672.

[14] The NRG Ljubljana package is avaiable at http://nrgljubljana.ijs.si.

[15] R. Zitko and Th. Pruschke, Phys. Rev. B 79 (2009) 085106.

[16] R. Zitko, Comp. Phys. Comm. 180 (2009) 1271. 